\title{
ANÁLISE E DISCRIMINAÇÃO DE QUIMIOTIPOS DE Lippia graveolens H.B.K. DA GUATEMALA POR MICROEXTRAÇÃO EM FASE SÓLIDA, CG-EM E ANÁLISE MULTIVARIADA
}

Juan Francisco Pérez Sabino*, Max Mérida Reyes e Christian Daniel Farfán Barrera

Escuela de Química, Universidad de San Carlos de Guatemala, Edificio T-13, Ciudad Universitaria zona 12, Guatemala, 01012, Guatemala

Antonio Jorge Ribeiro da Silva

Núcleo de Pesquisas de Produtos Naturais, Centro de Ciências da Saúde, Universidade Federal do Rio de Janeiro, Bl. H, 21941902 Rio de Janeiro - RJ, Brasil

Recebido em 14/2/11; aceito em 13/6/11; publicado na web em 16/8/11

\begin{abstract}
ANALYSIS AND DISCRIMINATION OF THE CHEMOTYPES OF Lippia graveolens H.B.K. OF GUATEMALA BY SOLID PHASE MICROEXTRACTION, GC-MS AND MULTIVARIATE ANALYSIS. SPME-GC-MS, PCA and HCA multivariate techniques were used in order to evaluate their applicability to discriminate the three chemotypes (thymol, carvacrol and mixed) described for L. graveolens of Guatemala. The leaves of L. graveolens are used for treatment of colds, bronchitis, and as seasoning for food preparations, yielding essential oil up to $4.34 \%$. Leaves of 35 individuals from eight populations, and eight composite samples were analyzed using a DVB/Carboxen/PDMS fiber and GC-MS. PCA and HCA were carried out using eight markers ( $p$-cymene, cis-sabinene hydrate, linalool, terpinen-4-ol, thymol, carvacrol, (E)-caryophyllene and caryophyllene oxide). The three chemotypes of $L$. graveolens were satisfactorily discriminated.
\end{abstract}

Keywords: chemotype; Lippia graveolens HBK; SPME.

\section{INTRODUÇÃo}

L. graveolens, conhecida como orégano mexicano é reconhecida por possuir múltiplas propriedades medicinais, sendo amplamente usada na medicina tradicional, ${ }^{1}$ no tratamento de doenças gastrointestinais, respiratórias, reumatismo, câncer e tumores. ${ }^{2}$ As folhas aromáticas, secas ou frescas, são usadas na América Central como flavorizante de alimentos e são vendidas secas no mercado. ${ }^{3} \mathrm{Na}$ Guatemala encontra-se a planta em bosques secos e espinhosos subtropicais, em forma de arbustos finos de até $3 \mathrm{~m}$, nos departamentos de El Progreso, Chiquimula, El Petén e Zacapa. ${ }^{3}$ A planta não é cultivada em grande escala, sendo coletada em populações silvestres e vendida no mercado. ${ }^{2}$ As folhas da planta contêm alto teor de óleo essencial (até 4,34 \%), ${ }^{4}$ sendo coletadas em época de floração. Três quimiotipos, timol, carvacrol e misto, têm sido identificados em estudos sobre a variabilidade da composição do óleo de $L$. graveolens de cinco populações de regiões áridas e solo rochoso. ${ }^{5}$ Os óleos de L. graveolens da Guatemala têm mostrado atividade antimicrobiana importante contra bacterias Gram positivas e Gram negativas. ${ }^{6}$ No México, o óleo de L. berlandieri v. Shauer, sinonímia de L. graveolens de acordo com Pascual et al., ${ }^{1}$ apresentou conteúdo fenólico total de $151 \mathrm{mg}$ equivalentes a ácido gálico/mL e baixa atividade antioxidante pelo método do DPPH, embora a composição do óleo não tenha sido estudada. ${ }^{4}$

As técnicas de análise multivariada já mostraram sua utilidade para o estudo de problemas taxonômicos e de variabilidade intraespecífica em espécies com potencial econômico, em conjunto com estudos de distribuição geográfica. Assim, três quimiotipos foram encontrados no estudo da composição química dos óleos essenciais de Lippia alba da Colômbia após análise por cromatografia em fase gasosa acoplada à espectrometria de massas (CG-EM) e análise de componentes principais (PCA). ${ }^{7}$ Diferenças em populações dentro da mesma subespécie, entre subespécies e entre as

*e-mail: fpsabino@usac.edu.gt espécies Satureja montana e S. kataibelii, da Península Balcânica foram encontradas mediante a avaliação da composição dos óleos essenciais por PCA. ${ }^{8} \mathrm{O}$ polimorfismo químico dos óleos essenciais de Thymus caespetitius das Ilhas Açores, ${ }^{9,10}$ de T. pulegioides da Lituânia, ${ }^{11}$ de T. carnosus da Espanha ${ }^{12}$ e de Lychnophora ericoides do Cerrado brasileiro, foi demonstrado mediante a aplicação das técnicas de análise multivariada (PCA e HCA) à composição dos óleos. ${ }^{13}$ Alguns autores sugerem que o polimorfismo químico pode se dever à variabilidade genética ou à influência de fatores ambientais e edáficos. ${ }^{10,13}$

A microextração em fase sólida (MEFS ou Solid Phase Microextraction, SPME), técnica de extração não exaustiva, foi desenvolvida por Arthur e Pawliszyn no início dos anos $1990 .{ }^{14-16}$ A técnica apresentou utilidade para a classificação de quimiotipos de Matricaria recutita,${ }^{17}$ assim como na análise de substâncias voláteis de erva-mate (Ilex paraguariensis St. Hill), ${ }^{18}$ substâncias voláteis de plantas aromáticas, ${ }^{19}$ frações voláteis de alimentos, ${ }^{20}$ aromas de frutas ${ }^{21}$ e café, ${ }^{22}$ entre outros produtos naturais.

A análise de componentes principais (Principal Component Analysis, PCA) e a análise de agrupamento hierárquico (Hierarchical Cluster Analysis, HCA) são técnicas estatísticas multivariadas apropriadas para explicar as diferenças entre conjuntos de amostras caracterizadas por um número elevado de variáveis. ${ }^{23,24}$

No presente trabalho foi estudada a variabilidade intraespecífica de Lippia graveolens HBK de oito populações da Guatemala, utilizando MEFS e CG-EM, em combinação com PCA e HCA, para avaliar a aplicação deste método para a escolha de indivíduos de L. graveolens destinados ao cultivo e produção de óleos essenciais.

\section{PARTE EXPERIMENTAL}

\section{Coleta e preparação do material vegetal}

Folhas de 35 espécimes de L. graveolens H.B.K. foram coleta- 
das em julho de 2007 em oito populações de três departamentos da Guatemala. Exsicatas das amostras (registros: de 35.388 a 35.396) foram depositadas no Herbário USCG (Universidad de San Carlos de Guatemala). As localidades de coleta estão mostradas na Tabela 1. As amostras foram secadas à temperatura ambiente e uma fração foi separada e transportada ao Núcleo de Pesquisas de Produtos Naturais da UFRJ para análise por MEFS e CG-EM. O material vegetal foi moído e homogeneizado no laboratório. Oito amostras compostas foram preparadas a partir de amostras correspondentes a cada população.

\section{MEFS e análise por CG-EM}

O material proveniente de folhas secas e moídas de cada espécime de $L$. graveolens foi analisado individualmente. $\mathrm{O}$ mesmo procedimento foi utilizado para as amostras compostas. $60 \mathrm{mg}$ de material foram colocados em um recipiente de $4,0 \mathrm{~mL}$, que foi fechado hermeticamente com tampa especial de teflon. A amostra foi equilibrada a $80{ }^{\circ} \mathrm{C}$ por 5 min e submetida à extração por headspace por $15 \mathrm{~min}$ utilizando uma fibra composta por uma combinação de uma camada de $50 \mu \mathrm{m}$ de divinilbenzeno-polidimetilsiloxano (DVB-PDMS) sobre uma camada de $30 \mu \mathrm{m}$ de carboxen-polidimetilsiloxano (CcrboxenPDMS) abreviada como DVB/carboxen/PDMS e fornecida pela Supelco. Em seguida, a fibra foi dessorvida por $10 \mathrm{~min}$ a $260{ }^{\circ} \mathrm{C}$ no injetor do CG-EM.

A análise por CG-EM foi realizada em triplicata para cada amostra, em um cromatógrafo a gás acoplado a um espectrômetro de massas, Shimadzu GCMS-QP5000 utilizando-se as seguintes condições: coluna com fase estacionária HP5 (5\% fenil-metil-silicone) de $30 \mathrm{~m}$ x $0,2 \mathrm{~mm}$ e $0,25 \mu \mathrm{m}$ de espessura de fase. Programa de temperatura de 60 a $240{ }^{\circ} \mathrm{C}$ a $3{ }^{\circ} \mathrm{C} / \mathrm{min}$ e isoterma a $240{ }^{\circ} \mathrm{C}$ durante $7 \mathrm{~min}$. Injetor a $260{ }^{\circ} \mathrm{C}$ e interface a $200{ }^{\circ} \mathrm{C}$. Injeção no modo splitless. Condições do EM: ionização eletrônica a $70 \mathrm{eV}$, varredura de 40 a 700 Daltons. A identificação dos componentes voláteis foi feita utilizando-se seus espectros de massas e por comparação dos índices de retenção das substâncias com índices de retenção da literatura ${ }^{25}$ e com os obtidos da análise de padrões de referência (Sigma-Aldrich). ${ }^{26}$

\section{Análise multivariada da fração volátil de $L$. graveolens extraida por MEFS}

Os resultados cromatográficos da MEFS de L. graveolens foram submetidos à PCA e HCA, utilizando o programa XLSTAT 2008 (Addinsoft). Os dados submetidos à análise multivariada correspondem aos resultados da média calculada a partir das injeções em triplicata das frações voláteis das amostras extraídas por MEFS. Inicialmente a PCA foi realizada com 61 variáveis, correspondendo às 61 substâncias identificadas na fração volátil (resultados não mostrados). Posteriormente foram descartadas sucessivamente variáveis que apresentavam muitos valores nulos e outras varíaveis que contribuíam pouco na construção dos componentes principais. Finalmente, 8 variáveis foram selecionadas para realizar a PCA, sendo estas: $p$-cimeno, cis-hidrato de sabineno, linalol, terpinen-4ol, timol, carvacrol, $(E)$-cariofileno e óxido de cariofileno. O arranjo matricial consistiu de 43 linhas (número de amostras analisadas por grupo) e 8 colunas (variáveis ou substâncias selecionadas). Para realizar a PCA se utilizou a matriz de correlações de Pearson, obtendo-se gráficos para os dois primeiros componentes principais, para amostras e variáveis.

A HCA foi realizada utilizando as matrizes de 43 amostras e todas as variáveis (substâncias identificadas) e de 43 amostras e as 8 variáveis definidas na análise de PCA. O coeficente de dissimilaridade utilizado foi a distância euclidiana; para realizar o agrupamento foi utilizado o método pela associação média (unweighted pair-group average linkage). Foi escolhida a opção de truncamento automático para definir os conglomerados e obter o dendrograma.

\section{RESULTADOS E DISCUSSÃO}

A MEFS em modo headspace em combinação com CG-EM foi avaliada com o propósito de se estabelecer sua utilidade como ferramenta para a classificação ou discriminação rápida dos quimiotipos de L. graveolens. Após testes com material vegetal dos três quimiotipos de L. graveolens, a fibra de DVB/carboxen/PDMS foi escolhida por gerar um perfil cromatográfico similar aos obtidos com o óleo essencial dos três quimiotipos de L. graveolens. ${ }^{27}$ As amostras pertencentes ao quimiotipo misto (El Subinal, Magdalena, km 93,5) apresentaram uma fração volátil mais diversa, com um máximo de 74 substâncias detectadas, não tendo sido identificadas 26 substâncias, correspondendo a um máximo de 13,9\% de área relativa (Magdalena). Nas amostras pertencentes ao quimiotipo timol (El Oreganal, San José e Casas de Pinto) foram detectadas um máximo de 35 substâncias, das quais não foram identificadas 6 substâncias, equivalentes a um máximo de $8,0 \%$ de área relativa (Casas de Pinto). Entre as amostras pertencentes ao quimiotipo carvacrol (El Carrizal e La Tuna) foram detectadas 38 substâncias, não tendo sido identificadas 6 substâncias, correspondendo a um máximo de 5,8\% de área relativa (La Tuna).

A existência dos três quimiotipos de $L$. graveolens tinha sido demonstrada pela análise de óleos essenciais de cultivos do Instituto de Ciência e Tecnologia Agrícola (ICTA) da Guatemala. As amostras de óleo essencial foram obtidas da geração F1 de plantas cultivadas sob as mesmas condições, em um campo localizado em San Jerônimo ( $15^{\circ} 03^{\prime} 40^{\prime \prime} \mathrm{N}$; $90^{\circ} 15^{\prime} 00^{\prime \prime} \mathrm{O}$ ) a uma altitude de $1000 \mathrm{~m}$, no departamento de Baja Verapaz, partindo de sementes coletadas em populações previamente identificadas ${ }^{5}$ e novas populações localizadas nos departamentos de El Progreso, Zacapa e Chiquimula, ${ }^{27}$ mesmas populações que foram amostradas no presente estudo.

Tabela 1. Localização geográfica das populações de coleta das sementes de L. graveolens

\begin{tabular}{|c|c|c|c|c|c|c|}
\hline No. & Local & Município & Departamento & Latitude & Longitude & Altitude (m) \\
\hline 1 & Magdalena & San Agustín Ac. & El Progreso & $14^{\circ} 55^{\prime} 08^{\prime \prime}$ & $89^{\circ} 57^{\prime} 38^{\prime \prime}$ & 265 \\
\hline 2 & km 93,5 Rodoviária Interamericana & San Agustín Ac. & El Progreso & $14^{\circ} 55^{\prime} 34^{\prime \prime}$ & $89^{\circ} 56^{\prime} 48^{\prime \prime}$ & 278 \\
\hline 3 & El Subinal & El Progreso & El Progreso & $14^{\circ} 51^{\prime} 15^{\prime \prime}$ & $90^{\circ} 08^{\prime} 05^{\prime \prime}$ & 440 \\
\hline 4 & San José & Teculután & Zacapa & $14^{\circ} 59^{\prime} 12^{\prime \prime}$ & $89^{\circ} 41^{\prime} 49^{\prime \prime}$ & 222 \\
\hline 5 & El Oreganal & Teculután & Zacapa & $15^{\circ} 00^{\prime} 38^{\prime \prime}$ & $89^{\circ} 43^{\prime} 32^{\prime \prime}$ & 300 \\
\hline 6 & Casas de Pinto & Río Hondo & Zacapa & $15^{\circ} 01^{\prime} 25^{\prime \prime}$ & $89^{\circ} 36^{\prime} 35^{\prime \prime}$ & 180 \\
\hline 7 & El Carrizal & San Jacinto & Chiquimula & $14^{\circ} 37^{\prime} 18^{\prime \prime}$ & $89^{\circ} 28^{\prime} 58^{\prime \prime}$ & 700 \\
\hline 8 & La Tuna & Ipala & Chiquimula & $14^{\circ} 33^{\prime} 43^{\prime \prime}$ & $89^{\circ} 34^{\prime} 52^{\prime \prime}$ & 900 \\
\hline
\end{tabular}


A Tabela 2 apresenta os resultados da análise por MEFS-CG-EM das frações voláteis de L. graveolens das 8 localidades e das amostras compostas de cada localidade. As localidades estão em ordem descendente de oeste a leste na Tabela. As 5 amostras individuais e a amostra composta de El Subinal corresponderam ao quimiotipo misto, com conteúdos muito baixos de timol e sem a presença do carvacrol. Quatro amostras de espécimes de Magdalena correspondem ao quimiotipo misto e uma ao quimiotipo timol. A amostra composta foi preparada sem material da amostra M3, por insuficiência de material. Três amostras do local no km 93,5 da
Rodovia Interamericana corresponderam ao quimiotipo misto e duas ao quimiotipo timol (timol: 73,1-74,9\%), com predominância do quimiotipo misto na amostra composta. Nas localidades El Oreganal, San José e Casas de Pinto, todas as amostras de espécimes de L. graveolens (13) e as 3 amostras compostas, corresponderam ao quimiotipo timol (timol: 67,4-77,5\%). Das amostras do local El Carrizal 3 corresponderam ao quimiotipo carvacrol (carvacrol: 50,3$51,8 \%$ ) e uma ao quimiotipo timol (timol: 69,6\%). A amostra composta de El Carrizal foi preparada só com material dos espécimes correspondentes ao tipo carvacrol. As 3 amostras de espécimes do

Tabela 2. Percentagem de área das substâncias detectadas e identificadas por MEFS e CG-EM de L. graveolens e utilizadas como marcadores na análise multivariada

\begin{tabular}{|c|c|c|c|c|c|c|c|c|}
\hline Amostra & $p$-cimeno & $\begin{array}{c}\text { Hidrato de } \\
\text { cis-sabineno }\end{array}$ & linalol & Terpinen-4-ol & timol & carvacrol & $(E)$-cariofileno & $\begin{array}{c}\text { Óxido de } \\
\text { cariofileno }\end{array}$ \\
\hline IR & 1024 & 1070 & 1098 & 1178 & 1290 & 1299 & 1418 & 1580 \\
\hline $\mathrm{S} 1$ & 4,9 & 3,9 & 2,4 & 10,4 & --- & --- & 10,3 & 3,3 \\
\hline $\mathrm{S} 2$ & 4,1 & 5,3 & 1,8 & 9,7 & --- & --- & 12,3 & 3,7 \\
\hline $\mathrm{S} 3$ & 5,9 & 0,7 & 1,7 & 6,8 & 0,1 & --- & 12,3 & 4,4 \\
\hline $\mathrm{S} 4$ & 5,6 & 2,6 & 0,8 & 6,9 & 0,1 & --- & 4,0 & 2,7 \\
\hline $\mathrm{S} 5$ & 4,7 & 3,3 & 1,4 & 6,3 & 0,1 & --- & 6,6 & 2,9 \\
\hline SM & 4,2 & 3,0 & 1,9 & 8,7 & 0,1 & --- & 9,5 & 3,9 \\
\hline M1 & 8,1 & 1,9 & 1,7 & 9,3 & 0,2 & 0,4 & 10,4 & 4,7 \\
\hline M2 & 10,3 & 4,1 & 6,7 & 7,2 & 0,1 & 0,2 & 6,1 & 2,7 \\
\hline M3 & 1,3 & 0,1 & 0,3 & 0,2 & 74,9 & 0,3 & 0,5 & 1,0 \\
\hline M4 & 9,7 & 2,0 & 1,6 & 8,7 & 0,5 & 0,2 & 10,0 & 3,3 \\
\hline M5 & 6,6 & 3,9 & 8,5 & 6,8 & 0,4 & 0,1 & 5,6 & 3,1 \\
\hline MM & 8,1 & 1,9 & 1,7 & 9,3 & 0,2 & 0,4 & 10,4 & 4,7 \\
\hline K1 & 2,3 & 0,1 & 0,4 & 0,3 & 73,1 & 0,3 & 5,5 & 0,5 \\
\hline $\mathrm{K} 2$ & 6,1 & 4,5 & 5,4 & 7,1 & 1,6 & 0,1 & 8,6 & 3,6 \\
\hline $\mathrm{K} 3$ & 1,8 & --- & 0,1 & 0,2 & 74,9 & 0,2 & 4,8 & 0,8 \\
\hline K4 & 7,0 & 3,2 & 2,3 & 9,2 & 1,3 & 0,1 & 6,6 & 2,4 \\
\hline K5 & 11,5 & 3,6 & 2,1 & 8,9 & 0,4 & 0,2 & 5,6 & 2,8 \\
\hline KM & 1,2 & 1,7 & 1,4 & 3,5 & 37,3 & 0,2 & 5,7 & 2,7 \\
\hline O1 & 2,5 & 0,1 & 0,1 & 0,2 & 77,5 & 0,2 & 4,7 & 0,4 \\
\hline $\mathrm{O} 2$ & 3,1 & 0,1 & 0,1 & 0,1 & 72,6 & 0,3 & 6,0 & 0,8 \\
\hline $\mathrm{O} 3$ & 4,4 & --- & --- & 0,1 & 73,5 & 0,2 & 3,4 & 0,5 \\
\hline O4 & 3,4 & --- & 0,1 & --- & 73,4 & 0,3 & 5,6 & 1,0 \\
\hline $\mathrm{OM}$ & 3,0 & 0,1 & 0,1 & 0,2 & 72,1 & 0,3 & 5,4 & 0,4 \\
\hline SJ1 & 2,1 & --- & --- & 0,1 & 75,8 & 0,3 & 5,4 & 0,7 \\
\hline $\mathrm{SJ} 2$ & 4,3 & 0,1 & 0,2 & 0,2 & 70,4 & 0,3 & 4,7 & 0,9 \\
\hline $\mathrm{SJ} 3$ & 4,2 & --- & 0,2 & 0,1 & 72,8 & 0,3 & 5,4 & 0,7 \\
\hline SJ4 & 4,3 & 0,1 & 0,2 & 0,2 & 71,4 & 0,3 & 9,0 & 0,6 \\
\hline SJM & 2,4 & 0,1 & 0,2 & 0,2 & 72,5 & 0,3 & 6,0 & 1,0 \\
\hline CP1 & 3,0 & --- & --- & 0,1 & 72,1 & 0,3 & 4,2 & 0,7 \\
\hline $\mathrm{CP} 2$ & 1,9 & --- & --- & --- & 73,5 & 0,2 & 6,9 & 0,6 \\
\hline CP3 & 4,0 & 0,1 & 0,1 & 0,1 & 70,7 & 0,3 & 5,7 & 1,0 \\
\hline $\mathrm{CP} 4$ & 2,9 & --- & 0,1 & 0,2 & 71,4 & 0,3 & 5,3 & 0,6 \\
\hline CP5 & 5,9 & --- & --- & 0,1 & 67,4 & 0,4 & 4,4 & 0,9 \\
\hline CPM & 3,1 & 0,1 & 0,1 & 0,2 & 71,0 & 0,4 & 5,4 & 0,8 \\
\hline EC1 & 4,6 & --- & 0,2 & 0,3 & 8,8 & 49,3 & 8,7 & 1,3 \\
\hline $\mathrm{EC} 2$ & 3,4 & --- & 0,1 & --- & 69,6 & 1,8 & 6,9 & 0,8 \\
\hline EC3 & 4,3 & --- & 0,2 & 0,2 & 9,9 & 51,8 & 7,2 & 0,5 \\
\hline EC4 & 6,4 & 0,1 & 0,5 & 0,3 & 10,5 & 50,3 & 8,3 & 0,8 \\
\hline ECM & 4,3 & --- & 0,2 & 0,2 & 9,9 & 51,8 & 7,2 & 0,5 \\
\hline $\mathrm{T} 1$ & 6,8 & --- & 0,2 & 0,3 & 8,9 & 48,7 & 6,7 & --- \\
\hline $\mathrm{T} 2$ & 5,7 & --- & 0,2 & 0,2 & 9,9 & 49,4 & 5,7 & --- \\
\hline $\mathrm{T} 3$ & 5,6 & 0,1 & 0,3 & 0,3 & 10,3 & 50,9 & 6,4 & --- \\
\hline $\mathrm{TM}$ & 8,0 & 0,1 & 0,3 & 0,4 & 8,9 & 49,0 & 1,2 & --- \\
\hline
\end{tabular}

IR: Índice de retenção; S: El Subinal; M: Magdalena; K: km 93,5; O: El Oreganal; SJ: San José: CP: Casas de Pinto; EC: El Carrizal; T: La Tuna. 
local La Tuna corresponderam ao quimiotipo carvacrol (carvacrol: 48,7-50,9\%; timol: 8,9-10,3\%), assim como a amostra composta (carvacrol: 49,0\%; timol: 8,9\%).

\section{Tendência geográfica}

As localidades de coleta encontram-se no bosque espinhoso subtropical (me-S), correspondendo às populações de El Subinal, Magdalena, km 93,5, El Oreganal, San José e Casas de Pinto, localizadas em ambos os lados do Rio Motagua que percorre esse bioma. As populações de El Carrizal e La Tuna encontram-se em transições do bosque seco subtropical (bs-S) e bosque úmido subtropical temperado (bh-S (t)). Alguns autores consideram a região compreendida pelo bosque espinhoso subtropical e bosque seco subtropical como pertencentes a um mesmo bioma chamado "chaparral espinhoso", caracterizado pela aridez e pelo tipo de vegetação. Este "chaparral espinhoso" está dividido em biotopos, um tipo "cactal" correspondente ao bosque espinhoso subtropical, apresentando cactos (gêneros Nopalia e Opuntia) e outras espécies xerofíticas. O biotopo "zarzal", correspondente ao bosque seco subtropical, apresenta leguminosas com espinhos dos gêneros Acacia e Mimosa. ${ }^{28}$ Os solos do biotopo "cactal", onde estão localizadas as populações tipos misto e timol, pertencem à classe dos solos de serpentinas e rochas associadas da depressão do Río Motagua. Os solos do biotopo "zarzal", onde estão localizadas as populações tipo carvacrol, pertencem à classe de solos de cinzas vulcánicas de elevações médias. ${ }^{28}$

As diferenças de bioma são responsáveis em parte pela diferenciação de quimiotipos das plantas. No caso de L. graveolens se observou que o quimiotipo misto predomina na parte ocidental da região de coleta. A parte central da região de coleta parece ser uma área de transição entre os quimiotipos misto e timol (Magdalena, km 93,5). El Oreganal, do lado norte do Rio Motagua, também tem mostrado esses dois quimiotipos, ${ }^{27} \mathrm{com}$ predominância do quimiotipo timol, embora neste trabalho tenham sido encontrados só indivíduos do quimiotipo timol nesse local. Já as populações de San José e Casas de Pinto, localizadas próximo a El Oreganal, do lado sul do Rio Motagua, apresentam o quimiotipo timol. As localidades a leste da região de coleta de L. graveolens (El Carrizal e La Tuna) no biotopo "zarzal" apresentaram o quimiotipo carvacrol predominantemente.

Avaliação dos resultados das análises por MEFS-CG-EM de $\boldsymbol{L}$. graveolens utilizando técnicas de análise multivariada

A fibra de DVB/carboxen/PDMS foi escolhida para analisar as amostras de espécimes de L. graveolens, por se obter com essa fibra perfis cromatográficos mais similares aos obtidos com os óleos essenciais dos três quimiotipos de L. graveolens. ${ }^{27}$ Os resultados encontrados com a fibra de DVB/carboxen/PDMS foram suficientes para discriminar entre os três quimiotipos.

Para completar a avaliação de MEFS para a discriminação de quimiotipos de L. graveolens, os resultados foram submetidos à PCA e HCA. As Figuras 1 e 2 mostram os resultados da análise multivariada dos resultados de MEFS-CG-EM de L. graveolens coletada em julho de 2007. A Figura 1 apresenta a plotagem dos escores considerando as amostras e as 8 substâncias marcadoras como únicas variáveis ( $p$-cimeno, cis-hidrato de sabineno, linalol, terpinen-4-ol, timol, carvacrol, $(E)$-cariofileno e óxido de cariofileno). O primeiro componente explica 58,68\% da variância e a segunda componente explica 19,03\% dessa variância. As amostras aparecem diferenciadas em três grupos, com exceção da amostra KM que corresponde à amostra composta do local km 93,5.
Observações (eixos F1 e F2: $77.72 \%$ )

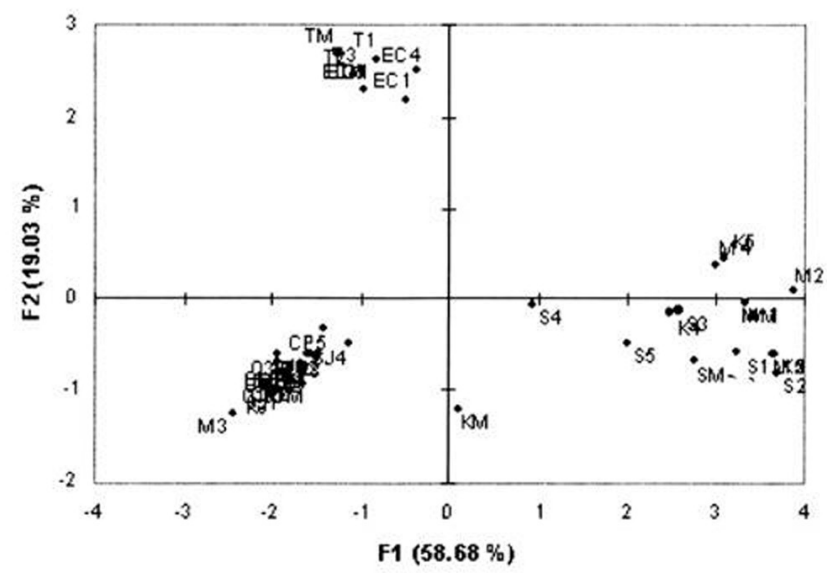

Figura 1. Gráfico dos escores das oito variáveis marcadoras e todas as amostras da ACP de MEFS-CG-EM de L. graveolens, coletada em julho de 2007

O isolamento da amostra KM significa que a presença de espécimes de mais de um quimiotipo em uma população pode levar a que uma amostra composta apresente componentes em percentagens que não permitam classificar a amostra como pertencente a um quimiotipo determinado. A PCA foi capaz de diferenciar os quimiotipos diferentes de uma mesma população, com exceção da amostra KM. A Figura 2 apresenta o dendrograma da HCA dos resultados de MEFS-CG-EM. Os três quimiotipos estão claramente diferenciados, o que confirma a utilidade de MEFS para discriminar os quimiotipos de L. graveolens. A amostra KM também aparece com diferença marcada do resto de amostras, sendo classificada como tipo timol.

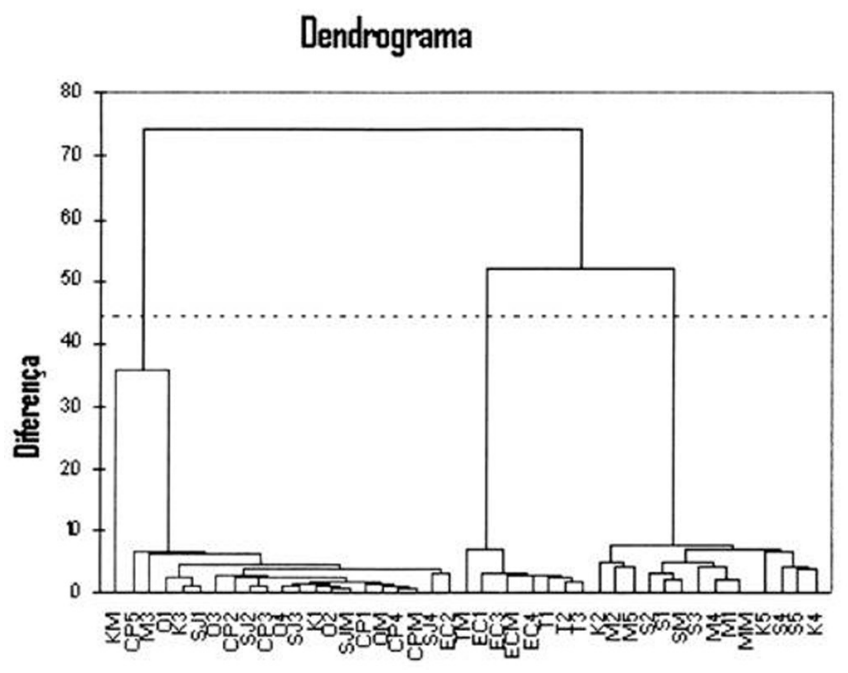

Figura 2. Dendrograma da AHC dos resultados de MEFS-CG-EM de espécimes de L. graveolens, coletados em julho de 2007

\section{CONCLUSÕES}

No presente estudo foi encontrada uma tendência na distribuição geográfica dos quimiotipos de L. graveolens, com predominância do quimiotipo misto no local mais ocidental da região de estudo (El Subinal), uma área de transição entre o quimiotipo misto e timol (localidades do centro da região de estudo), e as populações de San José e Casas de Pinto apresentando espécimes de tipo timol. O quimiotipo carvacrol apresentou predominância nas populações de El Carrizal e La Tuna, no leste da região de estudo. Considera-se necessário rea- 
lizar a coleta de um maior número de amostras e de populações para confirmar esta tendência. Na área de transição e em uma população com predominância de quimiotipo carvacrol (La Tuna), foram encontrados espécimes de $L$. graveolens correspondentes a mais de um quimiotipo. Isto nos permite deduzir que L. graveolens é uma planta que pode apresentar mais de um quimiotipo por população, o que é contrário às conclusões de estudos anteriores em que era presumido o quimiotipo partindo da concentração média de amostras compostas de vários espécimes.

A técnica MEFS apresentou como vantagem para seu uso na discriminação dos óleos essenciais de L. graveolens, menor tempo de extração em relação à hidrodestilação do óleo essencial, além de demandar menor quantidade de amostra (60 mg por MEFS contra 20 g de uma amostra típica para extração do óleo essencial). A MEFS pode, pelas razões acima, ser utilizada como ferramenta na seleção de espécimes de L. graveolens correspondentes a quimiotipos de interesse em uma população silvestre, para coleta de sementes e posterior cultivo da planta.

As técnicas de PCA e HCA mostraram ser úteis para diferenciação e agrupamento dos espécimes de L. graveolens nos três quimiotipos, em combinação com a MEFS, permitindo, assim, a rápida classificação de material de L. graveolens por quimiotipos.

\section{MATERIAL SUPLEMENTAR}

Um mapa de biomas com a localização dos sítios de coleta de $L$. graveolens encontra-se disponível em forma de arquivo PDF, com acesso livre, em

\section{AGRADECIMENTOS}

Ao NPPN-UFRJ pelo apoio nas análises por MEFS-CG-EM, ao $\mathrm{CNPq}$ pela bolsa de J. F. Pérez Sabino no programa PEC/PG, e à USAC da Guatemala, pelo apoio na coleta e preparação preliminar nas amostras.

\section{REFERÊNCIAS}

1. Pascual, M. E.; Slowing, K.; Carretero, E.; Sánchez Mata, D.; Villar, A.; J. Ethnopharmacol. 2001, 76, 201.

2. Hernández, T.; Canales, M.; Avila, J. G.; Duran, A.; Caballero, J.; Romo de Vivar, A.; Lira, R.; J. Ethnopharmacol. 2003, 88, 181; Cáceres, A.; Plantas de uso medicinal en Guatemala, Editorial Universitaria: Guatemala, 1998.

3. Standley, P. C.; Williams, L. O.; Gibson, D. N.; Flora of Guatemala, part IX, Fieldiana: Chicago, 1970.

4. Rocha-Guzmán, N. E.; Gallegos-Infante, J. A.; González-Laredo, R. F.; Ramos-Gómez, M.; Rodríguez-Muñoz, M. E.; Reynoso-Camacho, R.; Rocha-Uribe, A.; Roque-Rosales, M. R.; Food Chem. 2007, 102, 330.

5. Fischer, U.; Franz, Ch.; López, R.; Pöll, E. Em Proceedings of 27th International Symposium on Essential Oils; Franz, Ch.; Mathé, A.; Buchbauer, G., eds.; Allured Publ. Corp.: Illinois, 1997.

6. Salgueiro, L. R.; Cavaleiro, C.; Gonçalves, M. J.; Proença da Cunha, A.; Planta Med. 2003, 69, 80.
7. Durán, D. C.; Monsalve, L. A.; Martínez, J. R.; Stashenko, E. E.; Scientia et Technica 2007, 33, 435.

8. Slavkoska, V.; Jancic, R.; Bojovic, S.; Milosavljevic, S.; Djokovic, D.; Phytochemistry 2001, 57, 71.

9. Pereira, S. I.; Santos, P. A. G.; Barroso, J. G.; Figueiredo, A. C.; Pedro, L. G.; Salgueiro, L. R.; Deans, S. G.; Scheffer, J. J. C.; Phytochemistry 2000, 55, 241

10. Santos, P. A. G.; Barroso, J. G.; Figueiredo, A. C.; Pedro, L. G.; Salgueiro, L. R.; Fontinha, S. S.; Deans, S. G.; Scheffer, J. C.; Plant Sci. 2005, 169, 1112.

11. Loziene, K.; Vaiciuniene, J.; Venskutonis, P. R.; Biochem. Syst. Ecol. 2003, 31, 249.

12. Salgueiro, L.; Vila, R.; Tommas, X.; Tomi, F.; Cañigueral, S.; Casanova, J.; Proença, A.; Adzet, T.; Phytochemistry 1995, 38, 391.

13. Curado, M. A.; Oliveira, C. B. A.; Jesus, J. G.; Santos, S. C.; Seraphin, J. C.; Ferri, P. H.; Phytochemistry 2006, 67, 2363.

14. Arthur, C. L.; Pawliszyn, J.; Anal. Chem. 1990, 62, 2145.

15. Slack, G. C.; Snow, N. H.; Kou, D. Em Sample preparation Techniques in Analytical Chemistry; Mitra, S., ed.; Wiley Interscience: New Jersey, 2003, cap. 4.

16. Ulrich, S.; J. Chromatogr., A 2000, 902, 167; Zuba, D.; Parczewski, A.; Reichenbächer, M.; J. Chromatogr., B: Anal. Technol. Biomed. Life Sci. 2002, 773, 75 .

17. Rubiolo, P.; Belliardo, F.; Cordero, Ch.; Liberto, E.; Sgorbini, B.; Bicchi, C.; Phytochem. Anal. 2006, 17, 217.

18. Araújo Filho, H.; Tese de Doutorado, Universidade Federal do Rio de Janeiro, Brasil, 2005.

19. Bicchi, C.; Cordero, Ch.; Liberto, E.; Rubiolo, P.; Sgorbini, B.; Sandra, P.; J. Chromatogr., A 2005, 1071, 111; Bicchi, C.; Cordero, Ch.; Liberto, E.; Sgorbini, B.; Rubiolo, P.; J. Chromatogr., A 2007, 1152, 138; Richter, J.; Schellenberg, I.; Anal. Bioanal. Chem. 2007, 387, 2207; Jirovetz, L.; Buchbauer, G.; Ngassoum, M. B.; Geissler, M.; J. Chromatogr., A 2002, 976, 265.

20. Bicchi, C.; Cordero, Ch.; Liberto, E.; Rubiolo, P.; Sgorbini, B.; J. Chromatogr., A 2004, 1024, 217.

21. Augusto, F.; Valente, A. L. P.; Tada, E. S.; Rivellino, S. R.; J. Chromatogr, A 2000, 873, 117.

22. Bicchi, C. P.; Panero, O. M.; Pellegrino, G. M.; Vanni, A. C.; J. Agric. Food Chem. 1997, 45, 4680.

23. Brereton, R. G.; Chemometrics: Data Analysis for the Laboratory and Chemical Plant, John Wiley \& Sons, Ltd.: West Sussex, 2003.

24. de Sousa, R. A.; Borges Neto, W.; Poppi, R. J.; Baccan, N.; Cadore, S.; Quim. Nova 2006, 29, 654.

25. Adams, R. P.; Identification of Essential Oil Components by Gas Chromatography/Quadrupole Mass Spectroscopy, Allured Publ. Corp.: Illinois, 2001.

26. Aquino Neto, F. R.; Nunes, D. S. S.; Cromatografia. Princípios Básicos e Técnicas Afins, Interciência: Rio de Janeiro, 2003.

27. Pérez Sabino, J. F.; Tese de Doutorado, Universidade Federal do Rio de Janeiro, Brasil, 2008.

28. Villar, L.; La Flora Silvestre de Guatemala, Editorial Universitaria: Guatemala, 2008. 


\section{ANÁLISE E DISCRIMINAÇÃO DE QUIMIOTIPOS DE Lippia graveolens H.B.K. DA GUATEMALA POR MICROEXTRAÇÃO EM FASE SÓLIDA, CG-EM E ANÁLISE MULTIVARIADA}

\section{Juan Francisco Pérez Sabino*, Max Mérida Reyes e Christian Daniel Farfán Barrera}

Escuela de Química, Universidad de San Carlos de Guatemala, Edificio T-13, Ciudad Universitaria zona 12, Guatemala, 01012 , Guatemala

Antonio Jorge Ribeiro da Silva

Núcleo de Pesquisas de Produtos Naturais, Centro de Ciências da Saúde, Universidade Federal do Rio de Janeiro, B1. H, 21941 902 Rio de Janeiro - RJ, Brasil

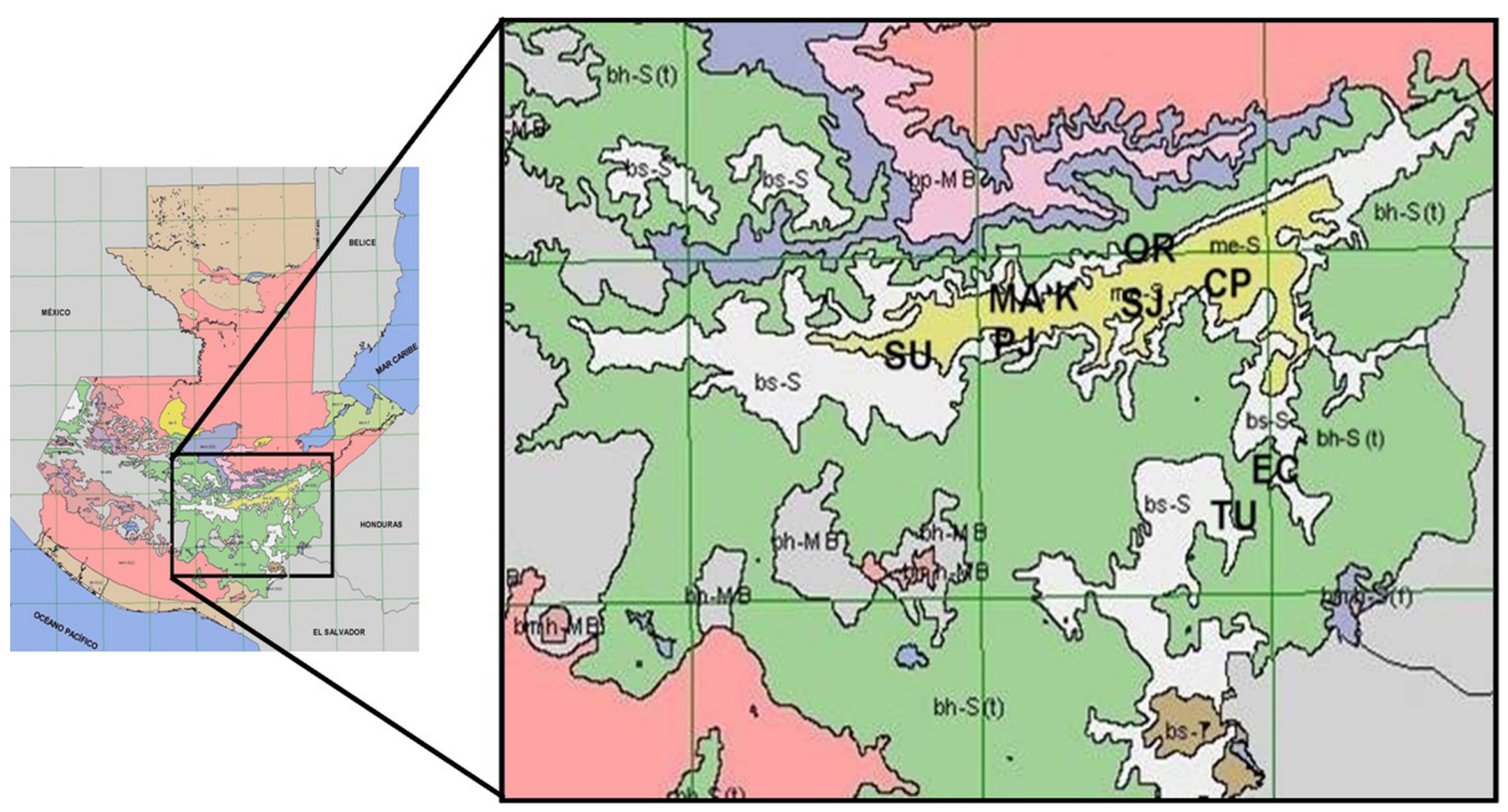

Figura 1S. Mapa de biomas da Guatemala com a região de coleta de L. graveolens delimitada e ampliada à direita. Biomas: me-S: Bosque Espinhoso Subtropical; bh-S (t): Bosque Úmido Subtropical (temperado); bs-S: Bosque Seco Subtropical. Locais: El Subinal (SU), Paso de los Jalapas (PJ), Magdalena (MA), km 93,5 (K); El Oreganal (OR), San José (SJ), Casas de Pinto (CP), El Carrizal (EC), La Tuna (TU). Fonte de informação e autorização cartográfica: Instituto Geográfico Nacional "Ing. Alfredo Obiols Gómez” (puede circular, Registro No. 279-2009) 